\title{
Les sources attosecondes
}

\author{
Fabrice CATOIRE, Ludovic QUINTARD, Ondrej HORT, Antoine DUBROUIL et Eric CONSTANT \\ Université de Bordeaux, CEA, CNRS UMR5107, \\ Centre Laser Intenses et Applications (CELIA), 351 cours de la libération, 33400 Talence
}

catoire@celia.u-bordeaux 1.fr

Les sources attosecondes sont maintenant une réalité. Elles ont ouvert le domaine de l'attoscience et permettent l'étude de dynamiques ultrarapides à des échelles de temps attoseconde et, en particulier, l'étude de dynamiques électroniques. Ces sources XUV ultracourtes sont aussi cohérentes et ont de nombreuses caractéristiques similaires au laser. Cet article présente le principe physique de leurs générations, leurs contrôles et leurs utilisations et montre ainsi comment la physique non-linéaire induite par laser femtoseconde intense permet aujourd'hui le développement de l'attophysique.

\section{Les sources attosecondes un outil idéal pour l'étude des dynamiques électroniques}

Les électrons sont des constituants fondamentaux de la matière mais, malgré leur omniprésence, leurs dynamiques restent paradoxalement mal connues. Ils sont si légers qu'ils évoluent extrêmement rapidement et des énergies de quelques électronvolts sont suffisantes pour leur conférer des vitesses proches de la célérité de la lumière (une énergie cinétique de 1 eV correspond à une vitesse qui est seulement 500 fois plus faible que celle de la lumière). Dans le modèle de Bohr, le temps de révolution sur la première orbite est ainsi de 150 as ( 1 as $=10^{-18}$ s soit un milliardième de milliardième de seconde). Les observer pendant leur évolution demande donc d'avoir accès à une résolution temporelle attoseconde. Les attosecondes sont maintenant accessibles même si cette échelle de temps paraît incroyablement petite puisque l'attoseconde est à la seconde ce que la seconde est à l'âge de l'univers. Les impulsions attosecondes apparaissent aujourd'hui comme un outil privilégié permettant d'accéder aux dynamiques électroniques et, a fortiori, à de nombreuses autres dynamiques induites par les électrons.

Les sources attosecondes qui sont aujourd'hui développées permettent en outre d'étudier de nombreux autres phénomènes car ces sources ont de multiples caractéristiques proches de celles des lasers et en particulier de très bonnes propriétés de cohérence. Le but de cet article est de présenter les principales caractéristiques de ces sources qui sont actuellement en plein essor.

\section{Les lasers ultracourts intenses}

Les développements de ces nouvelles sources découlent de la fiabilisation des sources lasers femtosecondes ( $1 \mathrm{fs}=10^{-15} \mathrm{~s}$ soit un millionième de milliardième de seconde) qui sont maintenant des « produits » commerciaux utilisables en routine. Leur cohérence spatiale, caractéristique commune à de nombreux lasers, permet de focaliser la lumière sur de très petites dimensions et d'obtenir de forts flux lumineux. Leur faible durée permet en plus de confiner temporellement l'énergie électromagnétique. La combinaison de ces deux propriétés permet d'obtenir des densités d'énergie lumineuses énormes qui peuvent amener la matière à interagir avec plusieurs photons simultanément. Ces interactions sont appelées non-linéaires et des processus comme l'absorption de plusieurs photons laser pour ensuite réémettre un photon plus énergétique sont possibles. Par conservation d'énergie, l'énergie du photon émis est égale à la somme des énergies de tous les photons absorbés. C'est ce processus appelé génération d'harmoniques d'ordres élevés (GHOE) qui est à la base de la génération des impulsions attosecondes. Ces impulsions ultrabrèves sont obtenues en utilisant des radiations de forte énergie et de grande largeur spectrale.

La largeur spectrale définit le contenu en fréquence (ou en longueur d'onde) d'une source et une largeur spectrale importante est une caractéristique première des sources ultra-brèves. Réciproquement, une source monochromatique qui a un spectre étroit est forcément une source de longue durée. La même chose a lieu en musique : lorsqu'une note est frappée, il est difficile de définir sa fréquence alors qu'une note tenue longtemps a une fréquence parfaitement définie. De manière plus quantitative, fréquence et durée sont reliées par une relation d'incertitude ; il n'est possible de connaître une fréquence $v_{0}$ avec une incertitude $\delta v$ que si elle est mesurée pendant un temps $\delta \tau$ suffisamment long. La durée, $\delta \tau_{0}$, d'une impulsion lumineuse limite intrinsèquement le temps de mesure utile et toute impulsion ultracourte a une largeur spectrale $\delta v$ qui vérifie $\delta v \delta \tau_{0} \geq$ cte (voir encadré 1 ).

En appliquant la relation d'incertitude au cas des impulsions attosecondes, il apparaît qu'une impulsion de 100 as a une 


\section{Impact du contenu spectral et de la phase spectrale sur le profil temporel des impulsions femtosecondes}

Évolution du champ électrique d'une impulsion laser en fonction du contenu de la phase spectrale (phase en fonction de la fréquence ou de l'énergie du photon) pour la même distribution d'énergie donnée par la figure du haut. Un spectre large est nécessaire pour obtenir des impulsions ultracourtes et la relation de phase spectrale définit le profil temporel des impulsions. Lorsque la phase spectrale est constante (ou linéaire) la durée de l'impulsion est minimale (figures de gauche). On remarque l'évolution du champ électrique (courbe bleue) par rapport à son enveloppe (courbe rouge) en fonction de la valeur de la phase $\left(\phi_{\text {cep }}\right)$. Dans le cas où la phase spectrale est quadratique, l'évolution du champ électrique est toute autre : la fréquence instantanée varie pendant l'impulsion et la durée de l'impulsion est augmentée (figures centrales). Enfin, lorsque la phase spectrale est aléatoire le champ est lui-même très fortement perturbé (figures de droites).

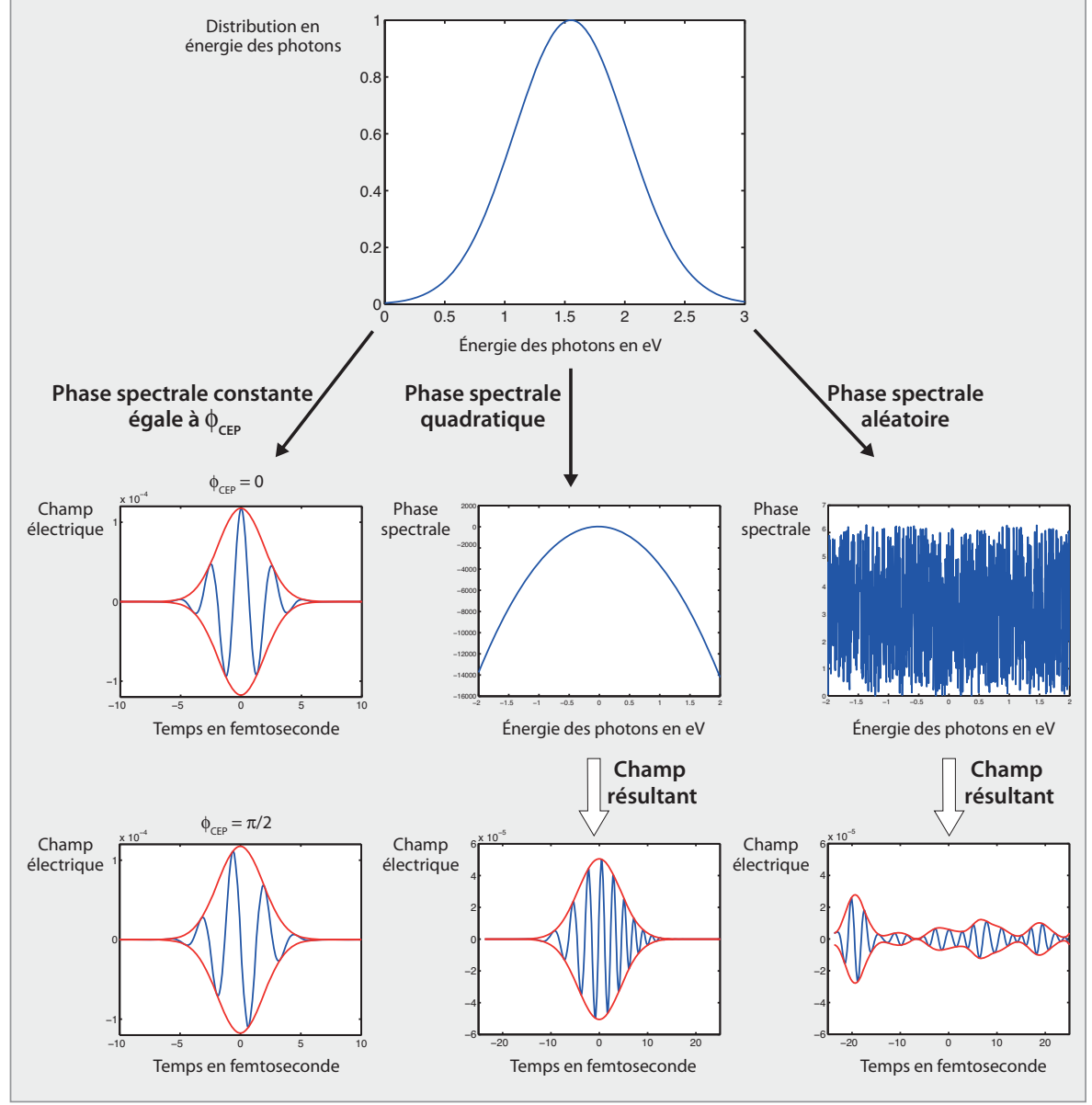

largeur spectrale supérieure à environ $5 \times 10^{15} \mathrm{~Hz}$ ce qui correspond à une distribution en énergie de photons de $20 \mathrm{eV}$ (pleine largeur à mi-hauteur). Le domaine visible est trop étroit pour une telle largeur spectrale car les photons du visible ont une énergie allant d'environ 1,5 eV (dans le rouge) à environ $3 \mathrm{eV}$ (dans le bleu). Ceci implique que des photons ayant une énergie d'au moins 20 eV sont nécessaires à la création d'impulsions attosecondes et que ces photons sont dans le domaine des XUV. Ces radiations XUV ultracourtes peuvent notamment être obtenues par le processus de génération d'harmoniques d'ordres élevés lors de l'interaction de lasers intenses avec des gaz ou des plasmas.

\section{Génération d'harmoniques d'ordres élevés}

La génération d'harmoniques d'ordres élevés est un processus non-linéaire qui

\section{acal $^{\text {bfi }}$}

European leader in advanced technology solutions

Votre Application $\downarrow$

Nos Lasers

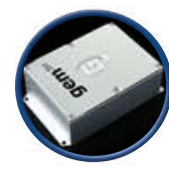

FINESSE

Laser de pompe $532 \mathrm{~nm}$

CEP feedback technology

ultra low noise

OPUS

Haute puissance à 532, 660 et $1064 \mathrm{~nm}$ dans un design compact GEM

Puissances et longueurs d'onde

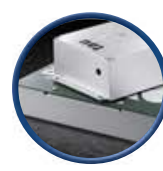
pour intégration, scellé hermétiquement $500000 \mathrm{H}$ MTBF

VENTUS

Puissances et longueurs d'ond pour applications scientifiques scellé hermétiquement $500000 \mathrm{H}$ MTBF TORUS Lasers monomodes longitudinaux Mode locking actif TAL

Lasers ultra-compacts pour intégration TACCOR

Lasers femtoseconde, $1 \mathrm{GHz}$ GIGAJET

Oscillateurs femtoseconde
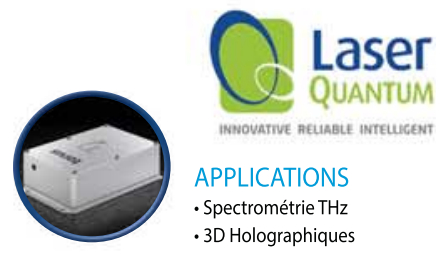

APPLICATIONS

- Spectrométrie $\mathrm{THz}$

-3D Holographiques

- Amplification d'impulsion

- Holographie

- Imagerie Biomédicale

- Imagerie par fluorescence

- Inspection de semiconducteurs

- Interférométrie

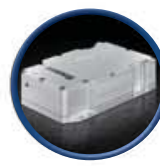

- Laser Doppler vélocimétrie (LDV)

- Lithographie

- Marquage et gravure

- Métrologie et peignes de fréquences

- Microscopie à deux photons et STED

- Ophtalmologie

- Pinces Optiques

- pompage de Ti:Sapphire

- Pompage optique de laser à colorants

- Pompage optique térahertz

- Séquençage d'A.D.N

- Spectroscopie Raman

- Spectroscopie à haute résolution domaine temporel

- Stabilisation d'enveloppe porteuse de phase (CEP)

- Triage de cellules

- Vélocimétrie par images de particules

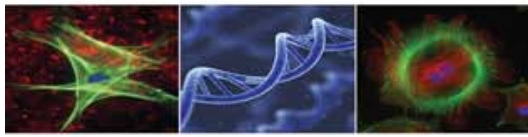

Pour plus d'informations

N'hésitez pas à solliciter nos ingénieurs Tel +33 (0)160795906 photonique.fr@acalbfi.fr

www.acalbfi.fr 
a lieu lorsqu'un laser intense (laser « fondamental » de période optique $T_{0}$ et de fréquence angulaire $\left.\omega_{0}=2 \pi / T_{0}\right)$ interagit avec un milieu et l'ionise. Ce processus conduit à l'absorption de $n$ photons à la fréquence fondamentale et à l'émission d'un photon $n$ fois plus énergétique de pulsation $\omega n=n \omega_{0}$. Ce processus est un processus cohérent et les symétries du milieu générateur alliées à la périodicité temporelle du processus d'émission impliquent que seules les fréquences harmoniques du fondamental sont émises. Pour un milieu générateur solide, la périodicité est $\mathrm{T}_{0}$ et toutes les harmoniques (paires et impaires) sont émises alors que pour un milieu gazeux, une anti périodicité $\mathrm{de}_{0} / 2$ implique que seules les harmoniques impaires sont émises de manière cohérente (figure 1).

Le nombre de photons absorbés n'est toutefois pas infini et cette énergie stockée (temporairement) par le système est quantifiée par une énergie appelée énergie pondéromotrice caractéristique du laser intense utilisé (voir encadré 2). Cette énergie pondéromotrice représente l'énergie cinétique moyenne d'un électron oscillant avec le champ électrique du laser intense. En effet, les lasers intenses sont couramment considérés comme de simples champs électriques oscillants ce qui est suffisant pour décrire l'essentiel de la physique sous-jacente. Cette énergie cinétique peut facilement atteindre des dizaines d'électronvolts lorsque le laser est suffisamment intense pour ioniser des atomes de gaz rares. Cette étape d'ionisation est indispensable à la GHOE car c'est la création d'électrons « libres » qui permet au système d'emmagasiner une fraction de l'énergie du laser qui est ensuite réémise sous forme de photons harmoniques.

Cette réémission XUV de l'énergie accumulée ne se fait qu'à des instants bien définis (au moment de la recombinaison dans le modèle à trois étapes) par rapport à l'échelle de temps typique du processus qui est la période optique, $T_{0}$, du fondamental. Cette période étant de l'ordre de $1 \mathrm{fs}$, les impulsions XUV sont émises sous la forme d'impulsions sub-femtosecondes. Lorsque les impulsions fondamentales sont longues et que la périodicité du processus est parfaite, l'émission des

\section{Encadré 2}

\section{Le modèle à trois étapes illustrant la GHOE dans les gaz}

Un des modèles les plus utilisés pour décrire la GHOE est appelé modèle à trois étapes (Corkum et al., 1994). Ce modèle est basé sur une description semi-classique de l'interaction laser intense / atome où la grandeur jouant un rôle fondamental est le champ électrique du laser et où l'atome est assimilé à un puits de potentiel où est localisé un électron. Ce modèle prend en compte trois étapes fondamentales que sont (1) l'ionisation de l'atome, (2) la propagation du paquet d'onde ionisé et enfin (3) la recombinaison.

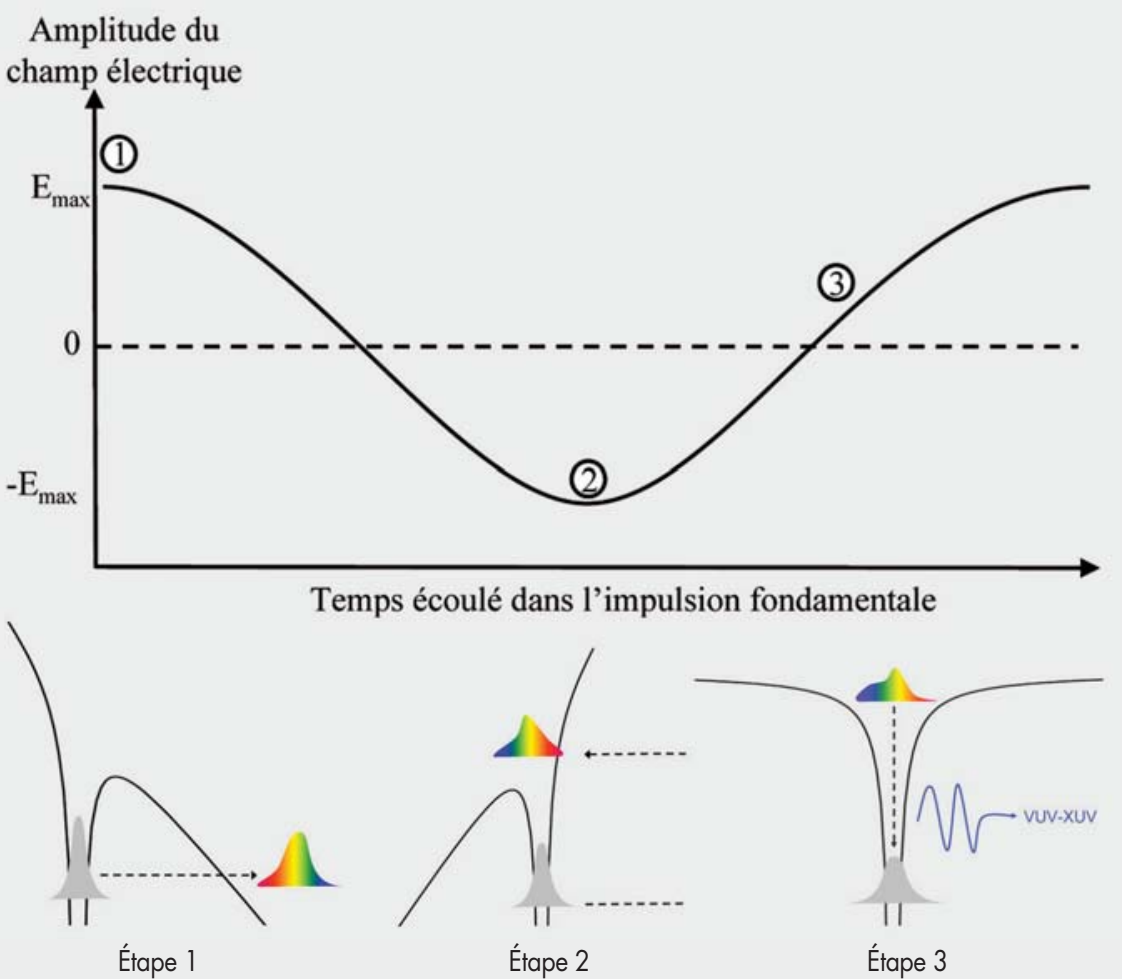

Étape 1 : Ionisation

L'ionisation de l'atome a lieu dans un régime où la barrière de potentiel vue par l'électron est abaissée en présence du champ laser ( $d$ 'intensité crête $I=E_{\max }{ }^{2} /(2 \times 377)$ et de fréquence centrale $\left.\omega\right)$. Une portion du paquet d'onde électronique lié (gris) est alors ionisée par effet tunnel (couleur).

\section{Étape 2 : Propagation}

Sous l'effet du champ laser oscillant, le paquet d'électron ionisé acquiert de l'énergie cinétique. L'énergie instantanée acquise lors de cette oscillation est proportionnelle à $U_{p}$ où $U_{p}=$

$$
\frac{q^{2} E_{\max }^{2}}{4 m \omega^{2}}
$$

est l'énergie pondératrice exprimée en unité atomique. $q$ et $m$ sont respectivement la charge et la masse de l'électron. Cette énergie est l'énergie moyenne d'oscillation d'un électron dans le champ laser.

\section{Étape 3 : Recombinaison}

Une portion du paquet d'onde libéré a une probabilité non nulle de revenir à proximité de l'ion parent. Une recombinaison vers l'état fondamental est alors possible en émettant un photon XUV. L'énergie maximale des électrons à ce moment est de $3,2 U_{p^{\prime}}$ donc l'énergie maximale des photons émis est de $3,2 U_{p}+I_{p}$ où $I_{p}$ est l'énergie d'ionisation.

impulsions XUV se fait sous la forme de trains d'impulsions attosecondes. Suivant les conditions utilisées (gamme spectrale sélectionnée, milieu générateur utilisé etc.), il peut y avoir une, deux ou quatre impulsions par période optique (figure 2). Ces trains d'impulsions attosecondes sont naturellement synchronisés 


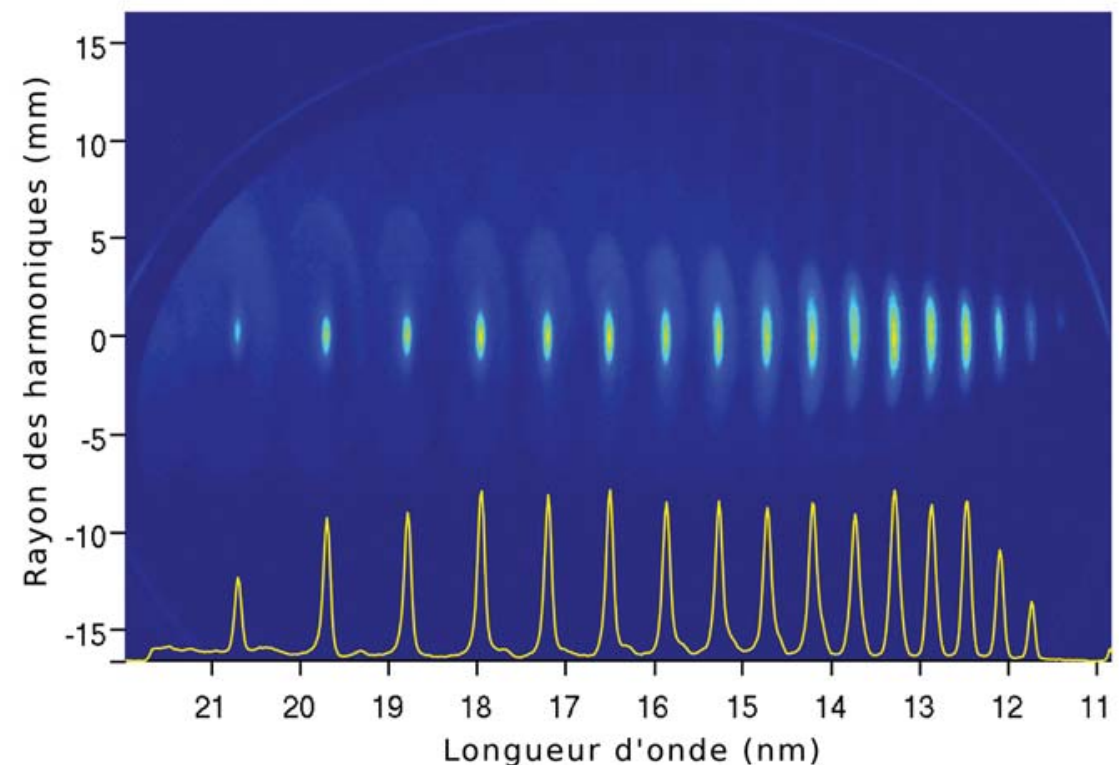

Figure 1. Spectre d'harmoniques d'ordres élevés généré dans un gaz de néon avec un laser femtoseconde intense. Ce spectre, résolu spatialement (axe des ordonnées), est constitué de pics harmoniques (harmoniques impaires du fondamental) et le profil spatial de ces faisceaux XUV est caractéristique d'une haute cohérence spatiale. On y voit des harmoniques ayant tous des amplitudes similaires dans un large domaine spectral (appelé plateau). Ce plateau se termine abruptement par une fréquence de coupure au-delà de laquelle la génération d'harmoniques est quasi inexistante. Malgré la faible efficacité du processus (l'énergie comprise dans chaque impulsion harmonique est typiquement $10^{-5}$ fois plus faible que l'énergie contenue dans l'impulsion fondamentale) les détecteurs actuels permettent d'enregistrer avec une haute précision des spectres comme celui-ci en quelques tirs laser.
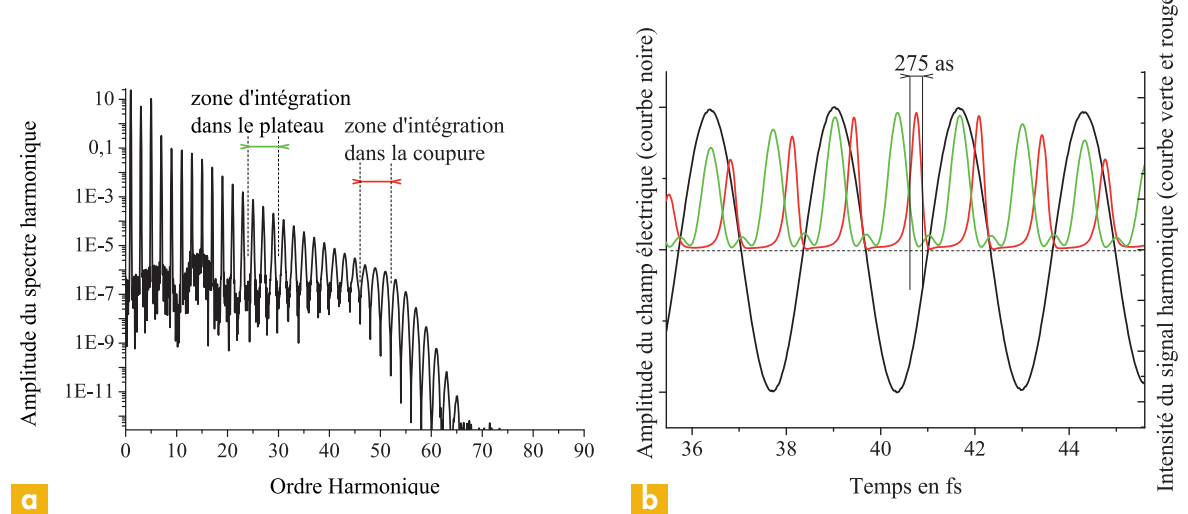

Figure 2. Spectre harmonique calculé (a) et profil temporel calculé (b) après avoir sélectionné des harmoniques du plateau (courbe verte) ou de la coupure (courbe rouge). Ces profils temporels sont constitués $\mathrm{d}^{\prime}$ impulsions attosecondes qui sont situées à des positions bien définies par rapport au champ électrique du laser fondamental (courbe noire).

avec le champ laser fondamental et les impulsions XUV peuvent initier des dynamiques ultra-rapides que l'on peut ensuite étudier en utilisant le laser comme un stroboscope attoseconde. Outre des dynamiques ultra-rapides, des grandeurs aussi fondamentales que le signe de fonctions d'ondes ou leur distribution deviennent accessibles (Remetter et al., 2006 ; Itatani et al., 2004).

Néanmoins pour réaliser des expériences à haute résolution temporelle, il est parfois préférable d'utiliser des impulsions attosecondes isolées. De nombreuses techniques ont été développées pour isoler ces impulsions attosecondes.

\section{$\triangle$ TRIOPIICS}

UN UNIVERS DE PRÉCISION

- Solutions complètes pour le test des systèmes optiques

- Interféromètres, goniomètres, collimateurs, autocollimateurs, bancs de tests, bancs de FTM.

- Mesure de focale, centrage, front d'onde, FTM, topographie..

- Applications en R \& D et production.

- Développements spéciaux.
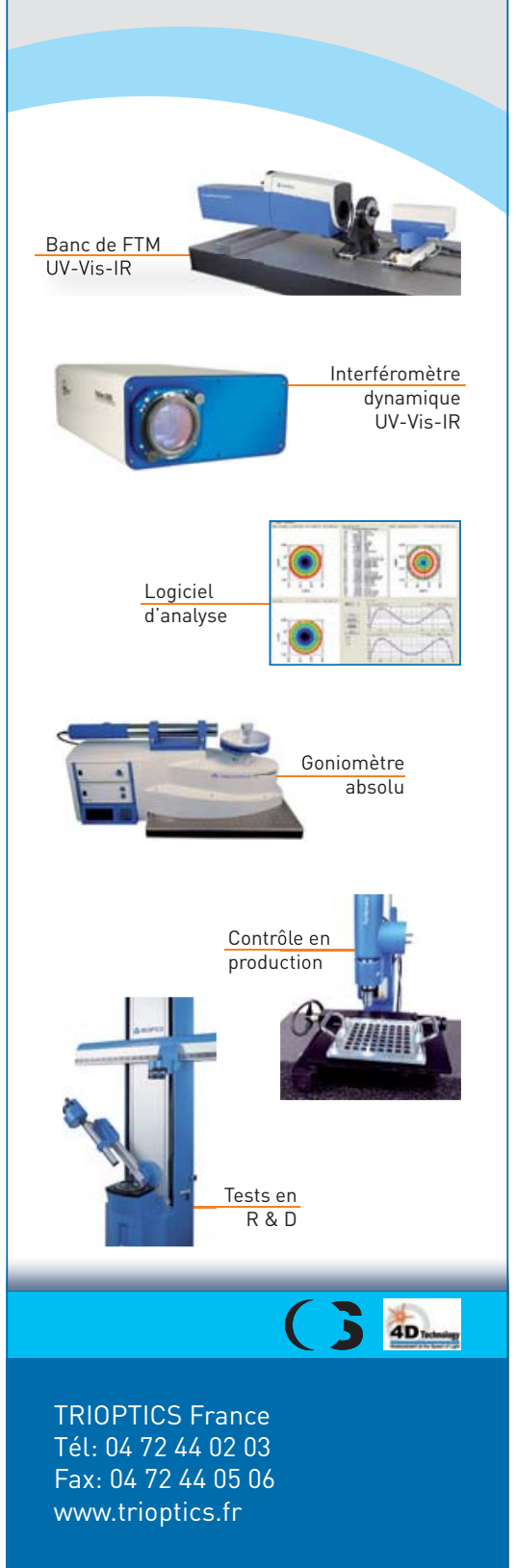

임 
Encadré 3

\section{Sélection d'une impulsion attoseconde isolée par modulation rapide de l'intensité}

Le processus de génération d'harmoniques est périodique en temps (voir encadré l) et est associé à l'émission d'un train d'impulsions brèves après sélection spectrale. Lorsqu'une source d'impulsions attosecondes isolées est désirée, un mécanisme permettant de sélectionner une impulsion unique est nécessaire. Cela peut être fait en brisant la périodicité du processus comme cela arrive si les impulsions fondamentales utilisées ont des durées de quelques cycles optiques seulement. Des exemples de spectres harmoniques (courbe noire en (b) et (d)) obtenus pour des impulsions fondamentales ultracourtes (respectivement associé aux courbes rouges (a) et (c)) sont présentés. Le profil temporel associé à l'émission des harmoniques de la coupure (au-delà de l'harmonique 23) est représenté par la courbe noire sur les figures (a) et (c). Pour certaines valeurs de la phase de la porteuse $\phi_{c e p}$ le spectre est structuré en harmoniques (d) et le profil d'émission XUV (c) est « long » alors que pour $d^{\prime}$ autres phases de porteuses une impulsion attoseconde isolée peut être générée (a) et le spectre de la coupure devient continu (b).
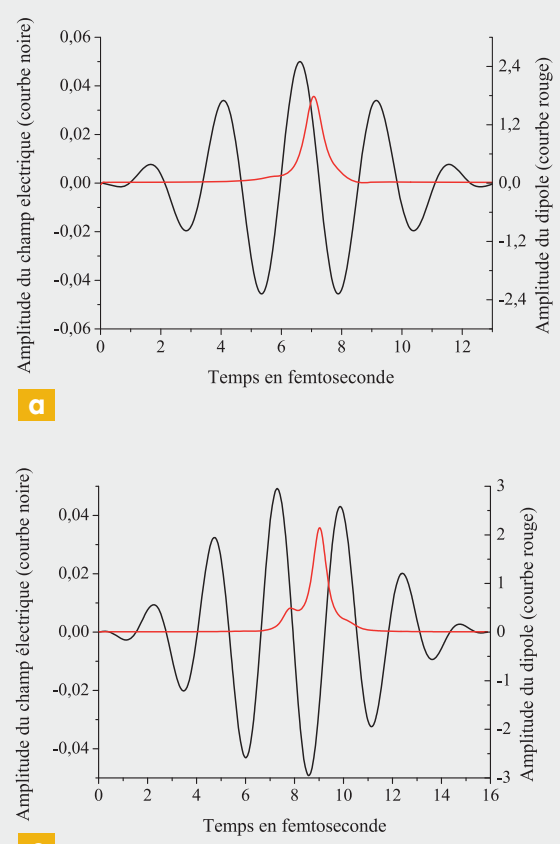

c

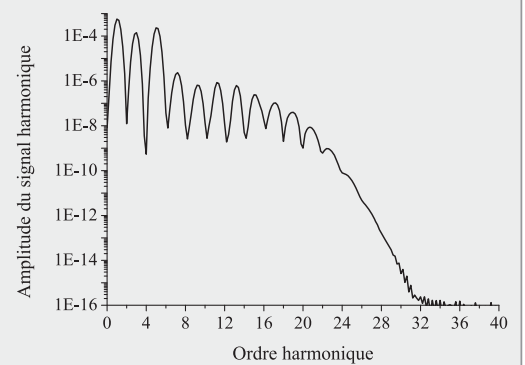

b

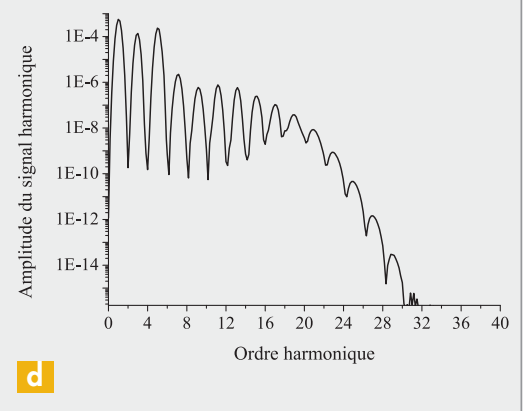

\section{Impulsions attosecondes isolées}

L'émission d'impulsions attosecondes sous forme de train lors de la GHOE est une conséquence de la périodicité du processus. Réduire la durée du train est possible en brisant cette périodicité et, à la limite, il est possible d'isoler une impulsion attoseconde unique en modulant très rapidement l'efficacité de la génération d'une période optique à la suivante. Lorsqu'une impulsion attoseconde isolée est sélectionnée, le spectre XUV devient continu et il est alors abusif de parler de génération d'harmoniques.

Moduler l'efficacité d'émission d'une période optique à l'autre est possible en jouant sur des paramètres qui influent grandement sur cette émission. Ces paramètres critiques (intensité, polarisation, taux d'ionisation etc.) sont nombreux dans la GHOE et, pour confiner l'émission, on peut utiliser:

- Une modulation du profil d'intensité des impulsions fondamentales (voir encadré3) en utilisant des impulsions fondamentales de 2-3 cycles optiques que l'on sait aujourd'hui produire. Ce fut la première approche réalisée expérimentalement (Hentschel et al., 2001).

- Une modulation de la polarisation du champ laser fondamental qui fut la première approche concrète proposée pour isoler une impulsion (Corkum et al., 1994).
- Une modulation de la densité d'émetteurs ou des conditions d'émission qui sont influencées par l'ionisation du milieu.

- Une modulation de la direction d'émission des impulsions qui, grâce à la cohérence spatiale du processus, peut être contrôlée en jouant sur le front de phase du fondamental (phare attoseconde) (Vincenti et Quéré, 2012).

- Des lasers fondamentaux multifréquences et utiliser les battements entre les différentes fréquences pour moduler l'émission. Cette approche a très récemment permis d'obtenir des impulsions attosecondes isolées avec des niveaux d'énergie proches du $\mu \mathrm{J}$ ce qui constitue le record actuel (Takahashi et al., 2013).

Ces différentes approches sont combinables entre elles à souhait et il existe donc de nombreuses techniques permettant de sélectionner des impulsions attosecondes isolées (Krausz et Ivanov, 2009). Leur contrôle nécessite toutefois une maîtrise parfaite des impulsions fondamentales (et notamment de leur CEP - carrier-envelope phase) et les développements actuels s'attachent à obtenir des techniques plus robustes et moins exigeantes sur les caractéristiques des impulsions fondamentales.

\section{Caractérisation et utilisation des impulsions altosecondes}

La caractérisation des impulsions est elle aussi très problématique, mais il existe maintenant de nombreuses techniques utilisées de par le monde (Streaking, TIGGER, FROG-CRAB, RABBITT etc. (Krausz et Ivanov, 2009)). Il reste néanmoins deux types d'approches qui donnent des résultats assez différents que ce soit pour des trains d'impulsions ou des impulsions isolées. Les mesures réalisées directement dans le domaine temporel par autocorrélation des impulsions attosecondes donnent généralement des impulsions dont les durées sont comprises entre 500 as et $1 \mathrm{fs}$. Les reconstructions réalisées à partir de mesures de phases spectrales donnent des durées qui peuvent être inférieures à la centaine d'attosecondes. Là encore, des développements 


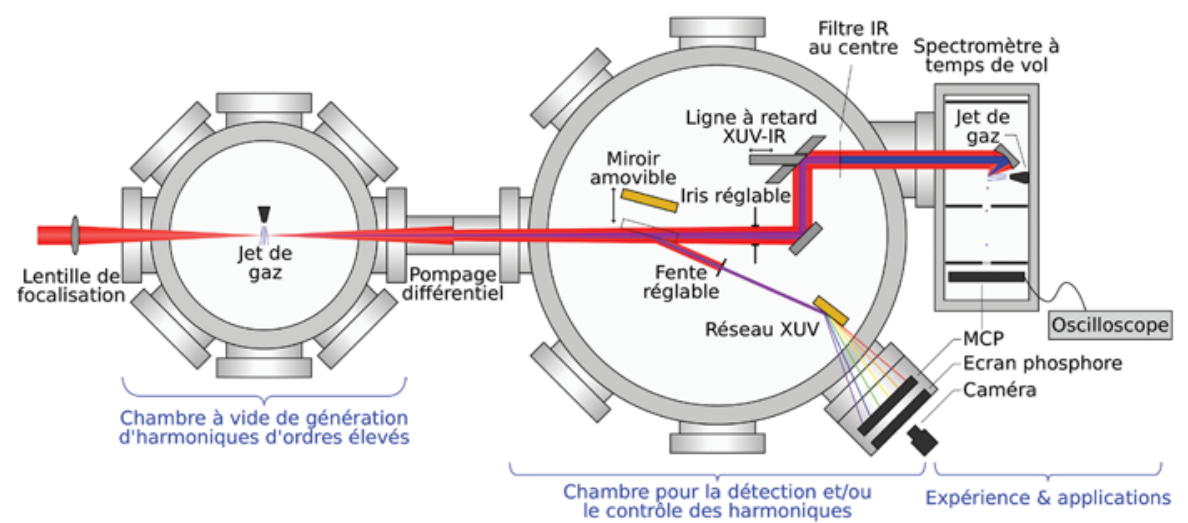

Figure 3. Ensemble expérimental typique permettant la génération d'harmoniques d'ordres élevés puis leur caractérisation et/ou contrôle et ensuite leur utilisation pour des expériences d'application.

sont en cours pour obtenir des valeurs similaires avec les différentes approches.

Les applications des impulsions attosecondes sont elles aussi en plein essor et bénéficient des nombreux progrès technologiques dans le domaine. Il devient maintenant possible avec des installations expérimentales compactes (figure 3) de générer des impulsions attosecondes puis de contrôler avec une précision attoseconde les délais entre ces impulsions et une fraction du champ laser fondamental qui leur a donné naissance. Il est alors possible d'initier un processus avec des impulsions attosecondes puis de le sonder avec le champ infrarouge (IR). Le délai XUV-IR introduit une échelle de temps qui permet de suivre l'évolution du processus étudié à l'intérieur d'une période optique du fondamental. De même, des transitions non-linéaires induites par impulsions attosecondes deviennent accessibles et permettent d'initier et de sonder des dynamiques après un temps d'évolution très bref. Des applications ont ainsi été réalisées dans des domaines très divers (Krausz et Ivanov, 2009) comme la physique atomique (suivi de cascades Auger, couplages entre niveaux etc.), la physique moléculaire (suivi de dissociation et de vibration, tomographie de fonction d'onde moléculaire) et la physique du solide (excitation et relaxation de solides, observation de niveaux profonds) ou même l'imagerie de nano-objets. Une des applications très prometteuses est le magnétisme à l'échelle attoseconde où des dynamiques de spin sont induites par des lasers ultrarapides dans des matériaux magnétisés. Cette thématique a déjà vu le jour dans le régime femtoseconde (stockage de données ultrarapide) et est à présent poursuivie vers des régimes attosecondes. Ces applications et la précision attoseconde qui est maintenant accessible ont par ailleurs conduit à des questionnements très fondamentaux qui portent par exemple sur la compréhension des liens non triviaux entre délais, temps et déphasages.

L'attophysique est un domaine qui jouit actuellement d'un grand développement au niveau mondial et qui bénéficie à la fois d'une bonne compréhension et de nombreuses avancées technologiques. Ce domaine a par ailleurs le privilège rare de tenir de nombreuses promesses qui paraissaient initialement illusoires et de continuer à ouvrir régulièrement de nouvelles perspectives tant appliquées que fondamentales.

\section{Références}

P.B. Corkum et al., Opt. Lett. 19, 1870 (1994).

M. Hentschel et al., Nature 414, 509 (2001).

J. Itatani et al., Nature 432, 867 (2004).

F. Krausz, M. Ivanov, Rev. Mod. Phys. 81, 000163 (2009)

T. Remetter et al., Nature Physics 2, 323 (2006).

E. Takahashi et al., Nature Comm. 4, 2691 (2013).

H. Vincenti, F. Quéré, Phys. Rev. Lett. 108, 113904 (2012).

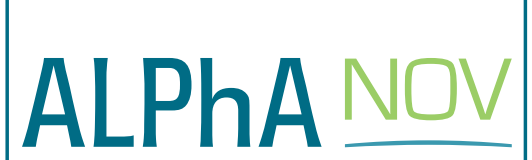

Centre Technologique Optique et Lasers

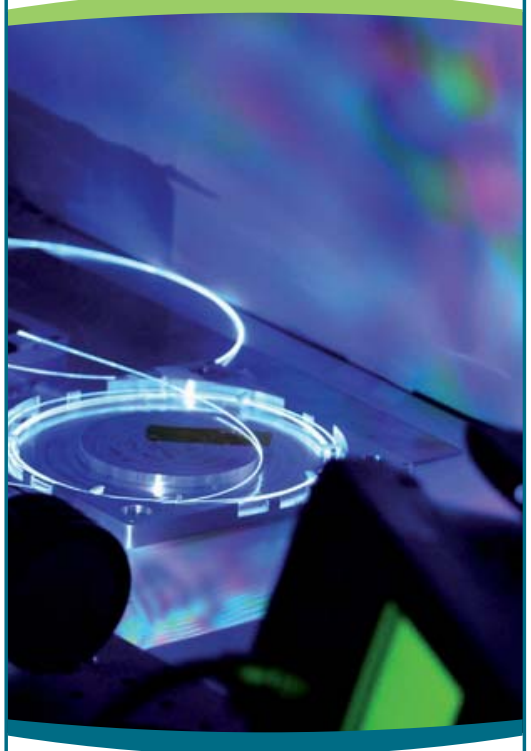

Votre partenaire en développements optiques

Nous vous accompagnons dans vos projets

- Sources lasers à fibres à façon

- Amplificateurs optiques haute performance

- Composants fibrés

- Interfaçage de fibres PCF

- Connecteurs sur fibres spéciales

- Micro-optiques...

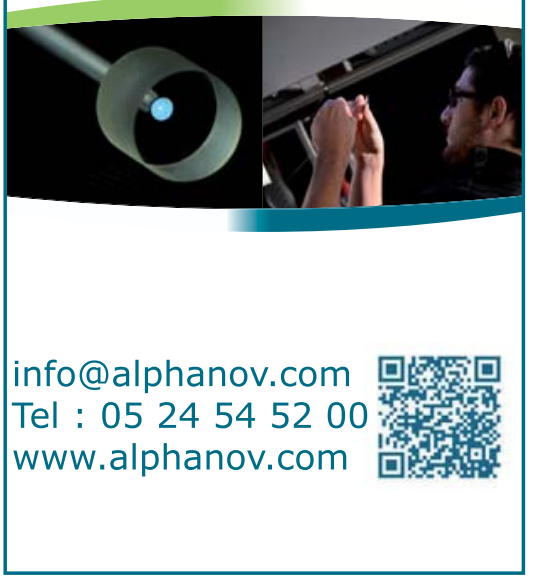

